\title{
Clinical significance of the histological and molecular characteristics of ependymal tumors: a single institution case series from China
}

Shaoyan $\mathrm{Xi}^{1+}$, Ke Sai ${ }^{2+}$, Wanming $\mathrm{Hu}^{1+}$, Fang Wang ${ }^{3}$, Yinsheng Chen ${ }^{2}$, Jing Wang ${ }^{2}$, Jing Zeng ${ }^{1 *}$ and Zhongping Chen ${ }^{2^{*}}$

\begin{abstract}
Background: Ependymal tumors are pathologically defined intrinsic neoplasms originating in the intracranial compartments or the spinal cord that affect both children and adults. The recently integrated classification of ependymomas based on both histological and molecular characteristics is capable of subgrouping patients with various prognoses. However, the application of histological and molecular markers in Chinese patients with ependymomas has rarely been reported. We aimed to demonstrate the significance of histological characteristics, the v-relavian reticuloendotheliosis viral oncogene homolog A (RELA) fusions and other molecular features in ependymal tumors.

Methods: We reviewed the histological characteristics of ependymal tumors using conventional pathological slides and investigate the RELA fusions and Cylclin D1 (CCND1) amplification by Fluorescence in situ hybridization (FISH) and trimethylation of histone 3 lysine 27 (H3K27me3) expression by immunohistochemistry (IHC) methods. SPSS software was used to analyze the data.

Results: We demonstrated that hypercellularity, atypia, microvascular proliferation, necrosis, mitosis, and an elevated Ki-67 index, were tightly associated with an advanced tumor grade. Tumor location, necrosis, mitosis and the Ki-67 index were related to the survival of the ependymomas, but Ki67 was the only independent prognostic factor. Additionally, RELA fusions, mostly presented in pediatric grade III intracranial ependymomas, indicated decreased survival times of patients, and closely related to the patients' age, tumor grade, cellularity, cellular atypia, necrosis and Ki67 index in the intracranial ependymal tumors, whereas reduction of H3K27me3 predicted the worse prognosis in ependymal tumors.
\end{abstract}

Conclusions: Histological and molecular features facilitate tumor grading and prognostic predictions for ependymal tumors in Chinese patients.

Keywords: Ependymal tumor, Histological characteristics, RELA, Prognosis, H3K27me3

\footnotetext{
* Correspondence: zengjing@sysucc.org.cn; chenzhp@sysucc.org.cn

'Shaoyan Xi, Ke Sai and Wanming Hu contributed equally to this work. 'Department of Pathology, Sun Yat-sen University Cancer Center, State Key Laboratory of Oncology in South China, Collaborative Innovation Center for Cancer Medicine, Guangzhou 510060, China

${ }^{2}$ Department of Neurosurgery/Neuro-oncology, Sun Yat-sen University

Cancer Center, State Key Laboratory of Oncology in South China,

Collaborative Innovation Center for Cancer Medicine, 651 Dongfeng Rd. East,

Guangzhou 510060, China

Full list of author information is available at the end of the article
}

(c) The Author(s). 2019 Open Access This article is distributed under the terms of the Creative Commons Attribution 4.0 International License (http://creativecommons.org/licenses/by/4.0/), which permits unrestricted use, distribution, and reproduction in any medium, provided you give appropriate credit to the original author(s) and the source, provide a link to the Creative Commons license, and indicate if changes were made. The Creative Commons Public Domain Dedication waiver (http://creativecommons.org/publicdomain/zero/1.0/) applies to the data made available in this article, unless otherwise stated. 


\section{Background}

Ependymomas are rare tumors originating from the ependymal epithelium throughout the neuroaxis and account for $3-10 \%$ of all neuroepithelial tumors. Although ependymomas can occur in patients at any age, children and young adults are prevalently affected. Ependymal tumors include sub-ependymomas, myxopapillary ependymomas and ependymomas. The prognoses of ependymal tumors are not identical and may depend on the extent of surgical resection, adjuvant therapy, and the molecular characteristics $[1,2]$. Classical ependymomas and anaplastic ependymomas correspond to the WHO grades II and III, whereas variants such as myxopapillary ependymomas or sub-ependymomas correspond to the WHO grade I tumors [3]. However, the criteria for grading ependymomas are not well established [4-6]. Few studies have investigated the prognostic significance of the WHO grade or individual pathological features across large trial cohorts. For the reasons above, the management of ependymomas is controversial due to uncertain biological behavior.

Genetic studies have shown that molecular alterations are very common in ependymomas, which display a broad range of cytogenetic aberrations [7, 8]. Ependymal tumors are divided into three subgroups (supratentorial, posterior fossa and spinal cord) according to the tumor location and nine molecular subgroups. One of the nine molecular alterations driving major prognostic implications is v-relavian reticuloendotheliosis viral oncogene homolog A (RELA)fusions [9]. RELA fusions often refer to as C11ORF95-RELA fusions. The RELA gene is located in 11q, and RELA fusions activate the Nuclear factor kappa B (NF-kB) cellular pathway, which is a central mediator of the cellular inflammatory response [10, 11]. The NF- $\mathrm{kB}$ signaling is observed in most human cancers. RELA fusions have been shown to activate the NF$\mathrm{\kappa B}$ target gene, upregulate L1 cell adhesion molecule (L1CAM) [2] and thereby have a profound impact on the expression of several other genes that regulate focal adhesion $[12,13]$. In a study by Mathew Parker et al. [9], the sequencing data of ependymomas cases showed amplification of CCND1in 18/89 cases. Additionally, since CCND1 is a direct transcriptional target of $N F-\kappa B$ signaling, it has the potential to contribute to the onset and/or progression of oncogenesis [14]. However, the significance of CCND1 amplification in ependymomas is not yet well studied.

Trimethylation of histone 3 lysine 27 (H3K27me3) is driven by aberrant DNA methylation and was used as a surrogate marker to distinguish between ependymomas posterior fossa group A (EP-PFA) and group B (EP-PFB) in a study of Panwalka [15]. H3K27me3 staining was found to be globally reduced in EP-PFA tumors, and immunohistochemistry (IHC) showed a sensitivity and specificity of $99 \%$ and $100 \%$, respectively, in segregating EP-PFA from EP-PFB tumors. H3K27me3 could be used to detect the DNA methylation of ependymomas in the routine practice as $\mathrm{IHC}$ is more convenience to perform than CpG island sequencing. H3K27me3 was also studied in high-grade gliomas and was found to have both diagnostic and biological significance. Decreased H3K27me3 was specific to a H3F3A K27 M mutant in Glioblastoma multiform (GBMs) while all other tumor subtypes, including H3F3A wild-type GBMs, showed strong H3K27me3 staining [16].

The combination of histological and molecular characteristics has pressing needs for deserving further research to improve the diagnosis and tailor treatment of ependymomas. In the current study, we investigated the histology and molecular markers of 69 ependymomas cases. We then performed Fluorescence in situ hybridization (FISH) to detect RELA gene fusions and explore the relationship between this variable and clinicopathological parameters. CCND1 was also examined by FISH to explore its prognostic value for ependymal tumor patients.H3K27me3 expression in ependymal tumors was also examined by using IHC and explored the prognostic value.

\section{Methods \\ Patients}

The data of patients received surgical treatment from January 2000 to December 2017 in our center and the

Table 1 The relationship between the pathological parameters and higher tumor grading

\begin{tabular}{|c|c|c|c|}
\hline & & 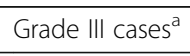 & $P$ value \\
\hline \multirow[t]{3}{*}{ Cellularity } & low & $6 / 15$ & 0.037 \\
\hline & moderate & $10 / 16$ & \\
\hline & high & $19 / 22$ & \\
\hline \multirow[t]{3}{*}{ Atypia } & mild & $8 / 18$ & 0.049 \\
\hline & moderate & $13 / 20$ & \\
\hline & severe & $13 / 15$ & \\
\hline \multirow[t]{2}{*}{ Necrosis } & present & $22 / 24$ & $<0.001$ \\
\hline & absent & $12 / 29$ & \\
\hline \multirow[t]{2}{*}{ Mitosis } & $\geq 5 / 2 \mathrm{~mm}^{2}$ & $18 / 21$ & 0.016 \\
\hline & $<5 / 2 \mathrm{~mm}^{2}$ & $16 / 32$ & \\
\hline \multirow[t]{2}{*}{ Vascular proliferation } & Present & 19/21 & 0.002 \\
\hline & Absent & $15 / 32$ & \\
\hline \multirow[t]{2}{*}{ Ki67 index } & $\geq 6 \%$ & $19 / 20$ & $<0.001$ \\
\hline & $<6 \%$ & $14 / 38$ & \\
\hline \multirow[t]{2}{*}{ RELA fusions } & Positive & $17 / 19$ & 0.001 \\
\hline & Negative & $20 / 48$ & \\
\hline
\end{tabular}

${ }^{a}$ : Some cases were lost due to inadequate tissue for testing or other reasons 

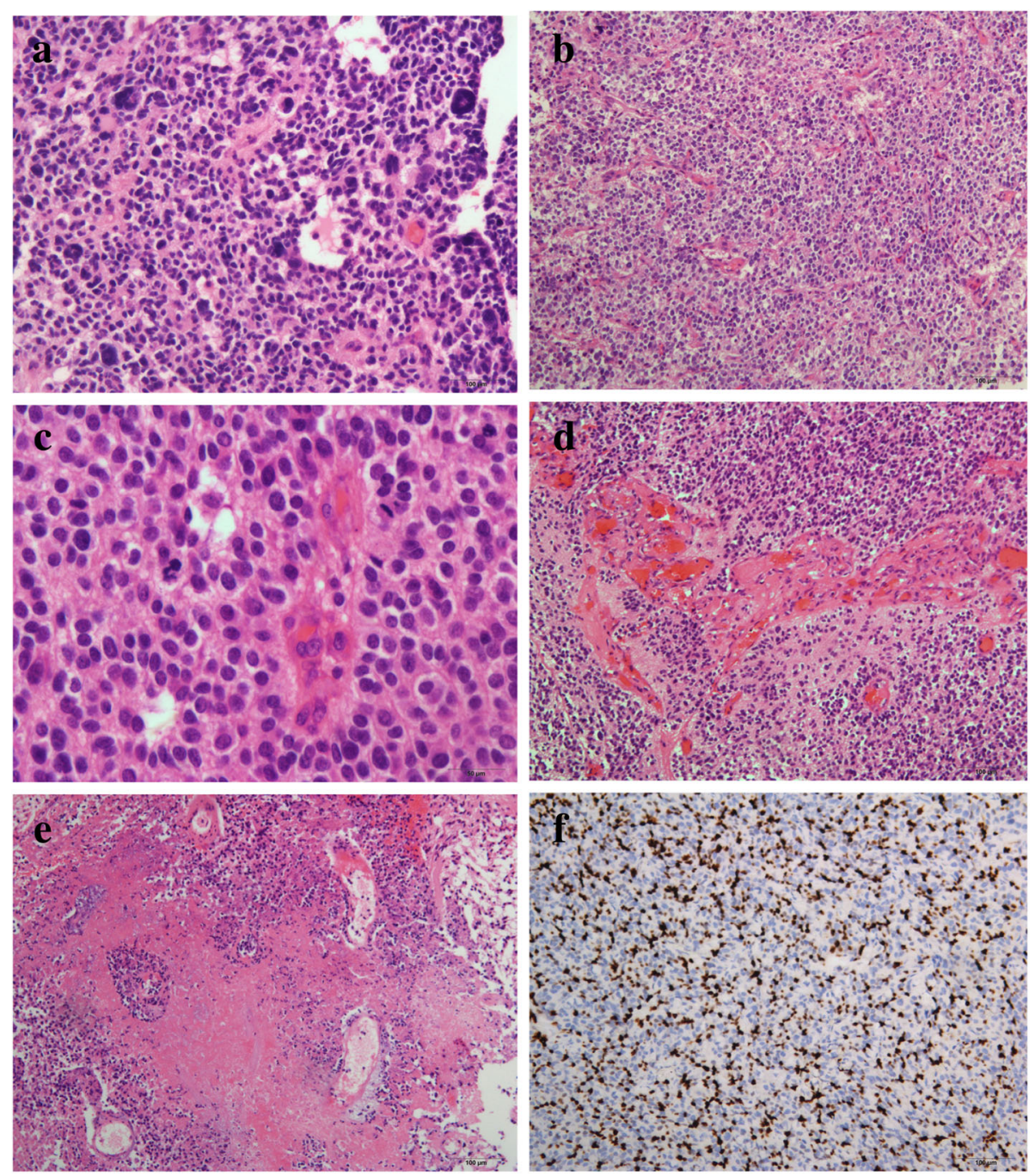

Fig. 1 (a) The figure shows pleomorphic tumor cells with nuclear atypia and multinucleated giant cells of the ependymal tumor; the bizarre nuclei were eye-catching; (b) The figure shows the high cellularity of the tumor, with crowding of the tumor cells; (c) The figure shows brisk mitosis in the tumors, with mitotic events counted in $2 \mathrm{~mm}^{2}$ high-power fields; (d) The figure shows microvascular proliferation, which often presents with a glomeruloid appearance; (e) The figure shows tumor necrosis, but palisading necrosis is easily seen; and (f) The figure shows the high Ki67 index in the ependymal tumors

surgical specimen diagnosed histologically as ependymal tumors were collected. Patients who receive therapy before surgery or died of diseases unrelated to ependymomas were excluded. A cohort of 69 cases was retrieved and this current study was approved by the ethics committee at our center. Written informed consent was obtained from all patients and/or their family members.

All cases were reviewed by two pathologists who were specialized in neuropathology. The histopathological characteristics evaluated comprised of cellularity, cellular atypia, necrosis, vascular proliferation, and mitosis.

\section{IHC staining}

IHC staining was performed following the standard procedures as briefly mentioned in one of our previous study [17]. Briefly, tissue slides were dewaxed and dehydrated according to our standard protocol. Antigen retrieval was performed by boiling the slides in antigen retrieval buffer for $15 \mathrm{~min}$. A primary antibody targeting Ki67 (DAKO, Denmark, MIB-1, Ready to use) and H3K27me3 (Abcam, Cambridge, UK, ab6002, 1:100) were added to the section and incubated at $4{ }^{\circ} \mathrm{C}$ overnight. A secondary antibody (DAKO, Denmark, ready to use) was added and incubated for $1 \mathrm{~h}$ after rinsing with PBS. DAB (DAKO, Denmark), was used for staining. H3K27me3 and Ki67 were positive for nucleus.

\section{FISH analysis}

FISH analysis was performed to detect the CCND1 gene copy number and RELA rearrangement status. 


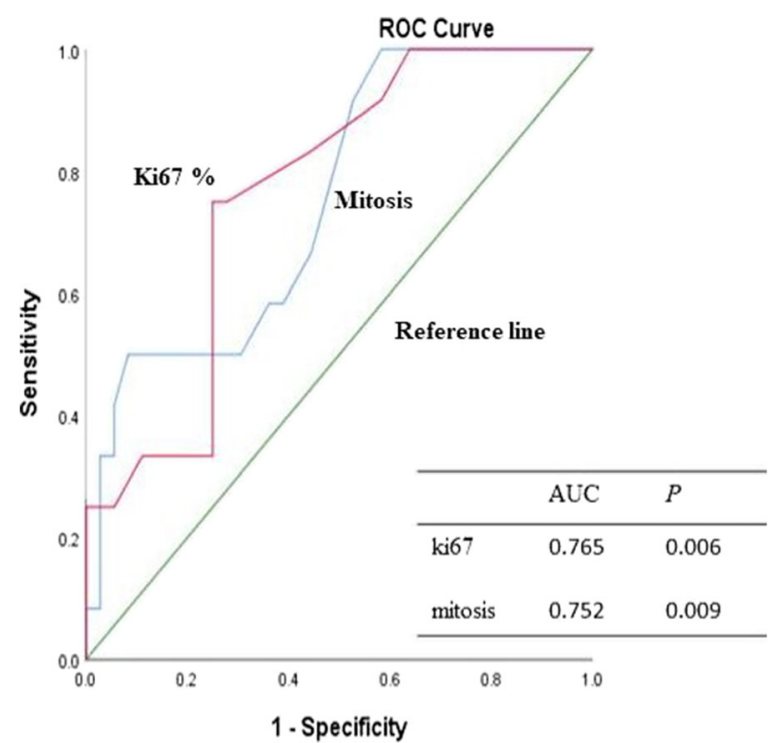

Fig. 2 ROC curve was used to identify the cutoff points for Ki67 and mitosis, with AUCs (areas under the curve) of 0.765 and 0.752 , respectively. The $P$ values were all $<0.05$. The cutoff points detected by the ROC curves for Ki67 and mitosis were $6 \%$ and $5 / 2 \mathrm{~mm}^{2}$, respectively

The FISH assay included the CCND1(11q13)/CSP11 probe and RELA (11q13) break apart rearrangement probe (LBP Medicine Science and Technology Co., Ltd., Guangzhou, China). Formalin-fixed, paraffin-embedded, 4- $\mu \mathrm{m}$-thick sections were used for FISH detection. The detailed FISH staining procedures was performed as we described previously. Hybridization signals for each probe were assessed under an Olympus BX51 TRF microscope (Olympus, Tokyo, Japan) equipped with a triple-pass filter (4',6'-diamidino-2-phenylindole, DAPI/Green/Orange; Abbott Molecular Inc., Des Plaines, IL, USA). The scoring was conducted in no less than 50 non-overlapping nuclei per core in tumor regions. Tumors were considered to be present for rearrangement if more than $15 \%$ of the nuclei demonstrated separate red and green signals. The average copy number of CCND1 and the chromosome11 centromere signal were determined, and a CCND1/CSP11 ratio was calculated for each case. Tumors with $\geq 4$ CCND1 signals per cell or a ratio $\geq 2.0$ were classified as CCND1 amplification.

\section{Statistical analysis}

The Statistical Product and Service Solutions (SPSS) statistical package for Window version 25 (SPSS, Chicago, IL, USA) was used for data analyses. The survival curves of ependymomas patients were derived with the Kaplan-Meier method. Multivariate analysis was performed by Cox regression. The correlation between clinical features of ependymomas patients and the tumor grade was analyzed using Pearson's $x^{2}$ test and ROC curves. The ROC curves were also used to identify the cutoff values for mitosis and the Ki67 index. A $P$ value $<0.05$ was considered to show statistical significance.

\section{Results}

Case cohort

A total of 69 patients were enrolled in the current study. Among them, they were 35 males and 34 females. The mean age at diagnosis was 25 years, and 26 patients were under 18 years old. Twenty-eight ependymomas originated in the supratentorial areas, 18 in the posterior fossa and 23 in the spinal cord. Thirty-eight, 29 and 2 cases were diagnosed as grade III, II and I, respectively. Total resection was achieved in 52 out of 69 cases. Forty-eight patients were treated with surgery only, 8 had plus radiotherapy, while 13 had surgery, followed by adjuvant radiotherapy and chemotherapy. The patients were followed up for 1 to 200 months. Eleven patients died from their tumors at the time of final follow-up.

\section{Histology-tumor grading}

All cases were reviewed by two pathologists who were specialized in neuropathology.. As shown in Table 1 and Fig. 1, cellularity, cellular atypia, vascular proliferation, necrosis, mitosis and the Ki67 index were associated with the tumor grade. Correlative analyse showed that RELA fusions were significantly related to the higher tumor grade because the most of RELA fusions events (16/17) occurred in grade III ependymal tumors. Additionally, the cutoff points for mitosis and Ki67 were $5 / 2 \mathrm{~mm}^{2}$ and $6 \%$, respectively, which was used to identify grade III tumors (Fig. 2). 


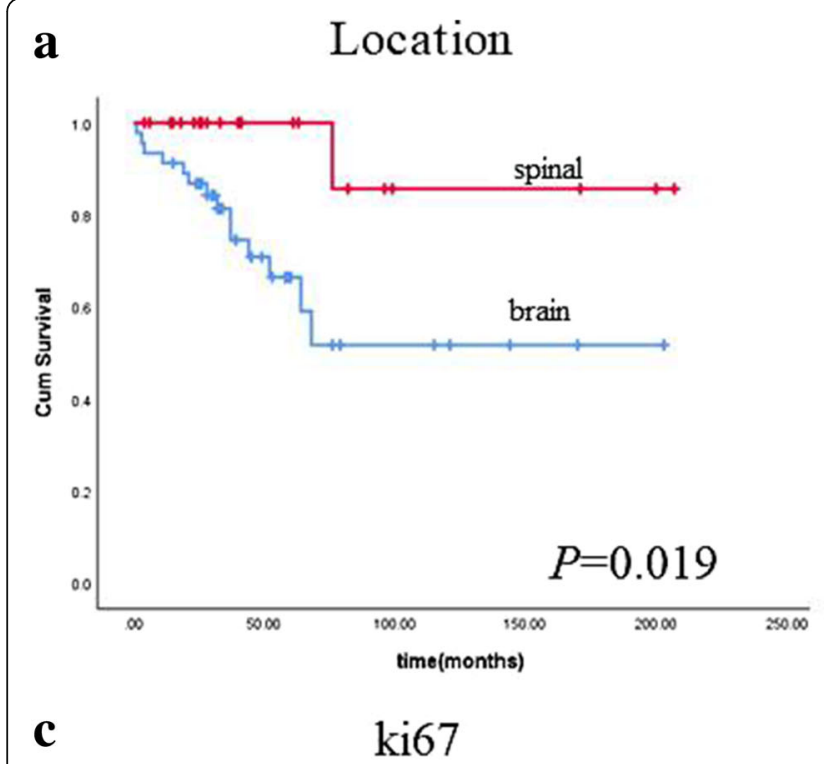

\section{b Necrosis}
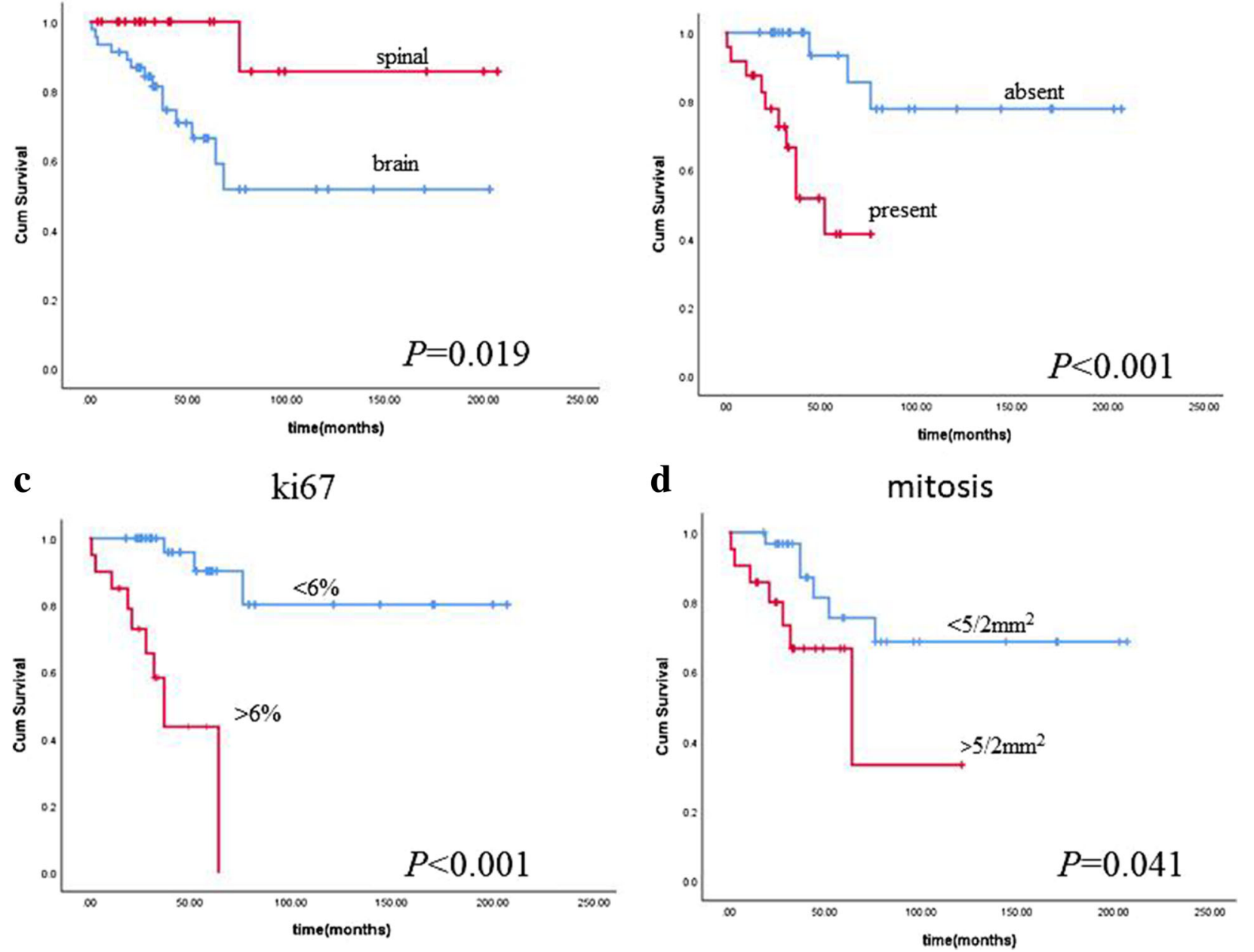

Fig. 3 Kaplan-Meier analysis showed that tumor location, necrosis, high Ki67 and high Ki67 mitotic activity were related to shorter survival times in ependymomas patients (a:124.06 \pm 17.31 months vs $188.29 \pm 17.31$ months, $P=0.019, \mathbf{b}: 174.82 \pm 16.47$ months vs $48.28 \pm 6.07$ months, $P<0.001$; c:179.05 \pm 15.76 months vs $40.92 \pm 6.35$ months, $P<0.001 ; \mathbf{d}: 157.03 \pm 17.36$ vs $67.58 \pm 15.88$ months, $P=0.041$; respectively)

Prognostic value of clinicopathological parameters in ependymal tumors

Univariable analyses were performed to investigate the prognostic factors related to survival. As shown in Fig. 3, the Kaplan-Meier analysis showed that tumor location (intracranial ependymomas or spinal ependymomas), necrosis, high Ki67and high mitotic activity were related to shorter survival times in ependymomas patients (124.06 \pm 17.31 months vs $188.29 \pm 17.31$ months, $P=$ $0.019 ; \quad 174.82 \pm 16.47$ months vs $48.28 \pm 6.07$ months, $P<0.001 ; 179.05 \pm 15.76$ monthsvs40.92 \pm 6.35 months, $P<0.001$; and $157.03 \pm 17.36$ vs $67.58 \pm 15.88$ months,

Table 2 Cox regression model to analyze the overall survival in ependymomas

\begin{tabular}{llll}
\hline Clinicopathologic & Multivariate analysis & & \\
\cline { 2 - 4 } features & Risk ratio & $95 \% \mathrm{Cl}$ & $0.279-2.891$ \\
\hline Tumor location & 0.898 & $0.000-3.995 \mathrm{E}+142$ & 0.941 \\
necrosis & $166,316.130$ & $0.128-2.071$ & 0.350 \\
mitosis & 0.515 & $1.158-62.958$ & $0.035^{*}$ \\
Ki67 index & 8.538 & &
\end{tabular}

$\mathrm{Cl}$, confidence interval, ${ }^{*} P<0.005$ represents statistically significant 
Table 3 Clinicopathological parameters correlated to RELA fusions in intracranial ependymal tumors

\begin{tabular}{|c|c|c|}
\hline & RELA fusions present & $P$ value \\
\hline Age & & 0.002 \\
\hline$\leq 18$ years & $13 / 21$ & \\
\hline$>18$ years & $4 / 24$ & \\
\hline \multicolumn{3}{|l|}{ Gender } \\
\hline Male & $11 / 25$ & 0.336 \\
\hline Female & $6 / 20$ & \\
\hline \multicolumn{3}{|l|}{ WHO grade } \\
\hline Grade II & $1 / 15$ & 0.008 \\
\hline Grade III & $16 / 30$ & \\
\hline \multicolumn{3}{|l|}{ Cellularity } \\
\hline low & $1 / 5$ & 0.047 \\
\hline moderate & $2 / 12$ & \\
\hline high & $11 / 19$ & \\
\hline \multicolumn{3}{|l|}{ Cellular atypia } \\
\hline mild & $1 / 9$ & 0.029 \\
\hline moderate & $4 / 13$ & \\
\hline severe & $9 / 14$ & \\
\hline \multicolumn{3}{|c|}{ Vascular proliferation } \\
\hline absent & $3 / 19$ & 0.003 \\
\hline present & $11 / 17$ & \\
\hline \multicolumn{3}{|l|}{ Mitosis } \\
\hline$\leq 5 / 2 \mathrm{~mm}^{2}$ & $4 / 19$ & 0.020 \\
\hline$>5 / 2 \mathrm{~mm}^{2}$ & $10 / 17$ & \\
\hline \multicolumn{3}{|l|}{ Necrosis } \\
\hline absent & $2 / 15$ & 0.008 \\
\hline present & $12 / 21$ & \\
\hline \multicolumn{3}{|l|}{ Ki67 index } \\
\hline$\leq 6 \%$ & $2 / 23$ & $<0.001$ \\
\hline$>6 \%$ & $12 / 16$ & \\
\hline \multicolumn{3}{|c|}{ CCND1 amplification } \\
\hline present & $5 / 14$ & 0.800 \\
\hline absent & $8 / 20$ & \\
\hline
\end{tabular}

*: Some cases were lost due to inadequate tissue for testing or other reasons

$P=0.041$;respectively). Cox regression analysis showed the Ki67 was the only independent biomarker for the prognosis in ependymal tumors (Table 2).

\section{RELA fusions related to clinicopathological parameters} and predicted a worse prognosis

RELA fusions were mostly present in intracranial ependymomas (17/19) and were significantly correlated with the age, tumor grade, cellularity, cellular atypia, necrosis and the Ki67 index in the supratentorial ependymal tumors (Table 3). RELA fusions were also significantly related to a shorter survival time in intracranial ependymomas $(P<0.001)$ (Fig. 4$)$.

\section{CCND1 amplifications in ependymal tumors}

There were $22(22 / 54)$ cases showing CCND1 amplification by FISH test. We also found that CCND1 amplification might predict a shorter survival $(159.44 \pm 20.12$ months vs $90.74 \pm 17.03$ months, $P=0.125)$. In further analyses, we found that the prognostic value of CCND1 amplifications in intracranial ependymomas was more distinct, although no statistical significance (Fig. 5).

The prognostic value of $\mathrm{H} 3 \mathrm{~K} 27 \mathrm{me} 3$ loss in ependymomas We performed IHC to evaluate H3K27me3 expression in ependymal tumors. H3K27me3 was absent in tumor cells but was preserved in endothelial cells and infiltrating lymphocytes in 25/58 cases. As shown in Fig. 6, the Kaplan-Meier analysis showed that a loss of H3K27me3 expression predicted a worsening prognosis $(56.12 \pm$ 6.67 months vs166.17 \pm 18.88 months, $P=0.011$, Fig. 6).

\section{Discussion}

The WHO system for grading of primary brain tumors including ependymomas can classify the low and high grade tumors as grades II or III, having survival time of $\geq 5$ years or $<5$ years, respectively [ 18 , 19]. The histological grade of ependymomas has been reported to be an independent prognostic indicator for event-free survival [3]. However, pathological parameters used for grading such as cell density, nuclear pleomorphism, mitotic activity and Ki67 index were prone to considerable interobserver variability, and not meet a consensus [20]. In our study, we proved that cellular atypia, vascular proliferation, necrosis and the Ki67 index were associated with tumor grade. As our experience, these pathological parameters reflected the activity of the tumor, indicating the tumor growth rate. Among the parameters, mitosis count and the Ki67 index were more objective and reproducible [21]. Mitotic activity and Ki67 was commonly used for the assessment of cell proliferation rate in virtually any field of tumor pathology [20]. Stefan Wolfsberger et al. used a Ki67 cutoff value of $20.5 \%$ to distinguish between grade II and grade III tumors [20],. However, in the experiences of usual pathological work, $20.5 \%$ is considered as a too high value for a cutoff point to differentiate between the grade II and III tumors. In the present study, we generated ROC curves for identification of cutoff points for the mitosis count and Ki67 index, found as were 5 per $2 \mathrm{~mm}^{2}$ and $6 \%$, respectively. The cutoff points that we made were more close to our usual work. Additionally, location, necrosis, mitosis and Ki67 index were related to 

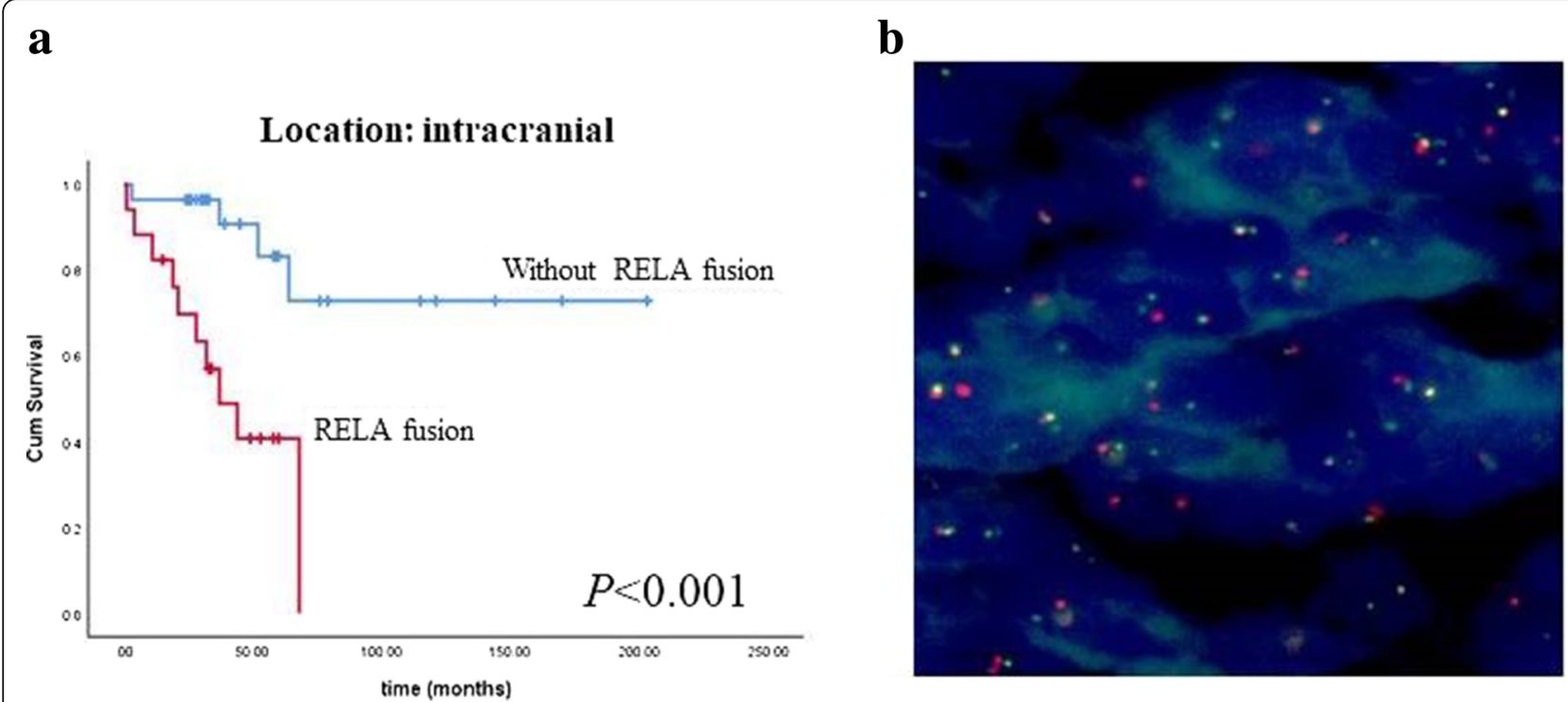

Fig. 4 Kaplan-Meier analysis showed that the RELA gene fusion group had shorter survival for intracranial ependymal tumors $(P<0.001)(\mathbf{a})$; RELA fusions were detected using the RELA break apart probe (b)

prognosis, but only Ki67 index was found to be an independent biomarker for survival. We demonstrated that the clinicopathological parameters were also significance for the prognostic prediction.

Parker et al. extensively demonstrated that C11orf95-RELA fusions resulted from chromothripsis of chromosome 11q based on whole-genome sequencing data and direct transcriptional targeting of NF$\kappa B$ signaling [9]. The NF- $\kappa B$ family of transcriptional regulators is central mediators of the cellular inflammatory response.[10] Therefore, the predictive value of RELA fusions for outcome attracted the attentions.
In the study of Marco Gessi [22], the authors failed to demonstrate the prognostic value of RELA fusions, while Figarella-Branger et al. reported RELA fusions predicted a better survival rate [23]. The finding of this present study showed that RELA fusions were related to a shorter survival time in intracranial tumors. The rate of RELA fusions accounting for ST-EPN was range 40 to $70 \%[9,22]$. In our series, most of the RELA fusions (17/19) occurred in intracranial ependymomas, $37.8 \%(17 / 45)$ of intracranial had RELA fusions, and they were related to a younger age, aggressive histopathological features. We firstly

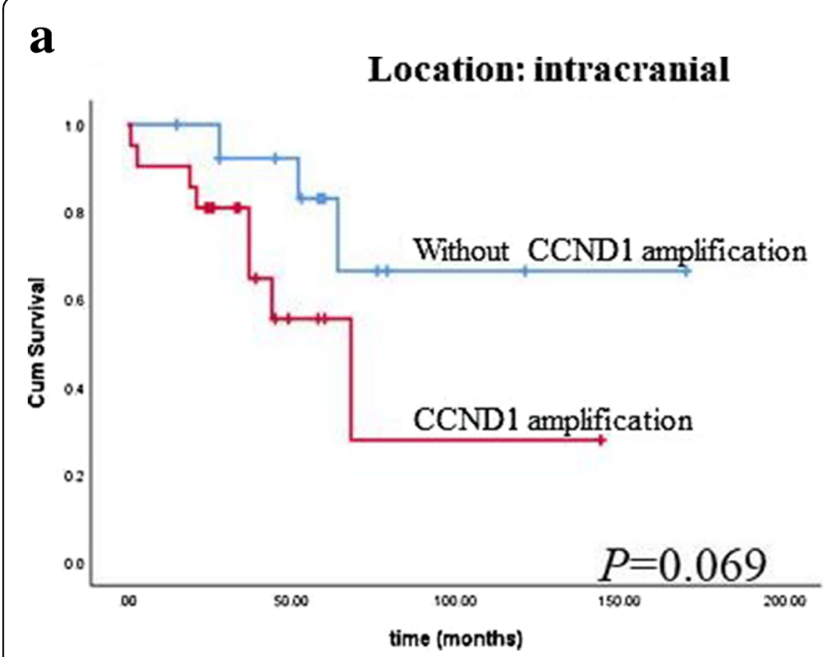

b

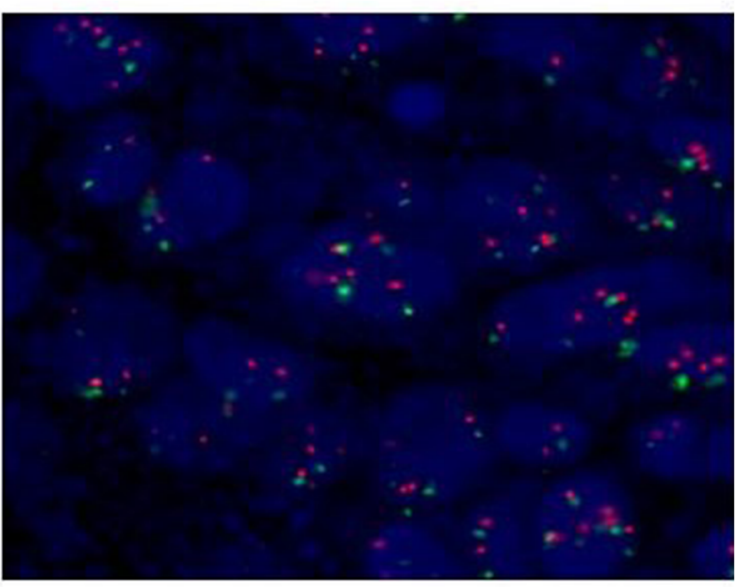

Fig. 5 Fig. 5 (a) Kaplan- Meier analysis showed that ependymal tumors with CCND1 amplifications had shorter survival in the intracranial ependymal tumors $(P=0.069)$ Figure $5 b$ showed that the CCND1 amplifications 


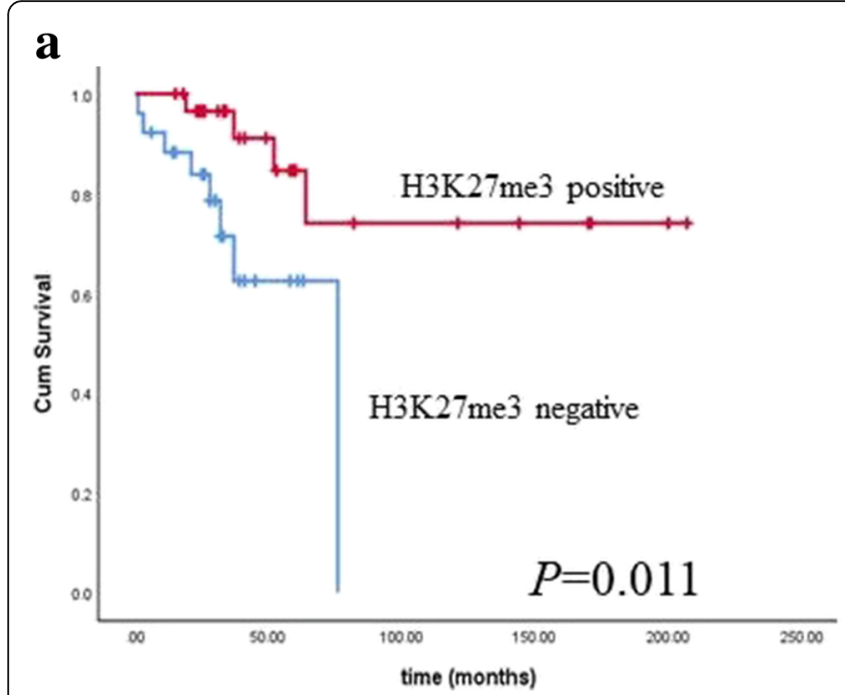

b

Fig. 6 Kaplan-Meier analysis showed that ependymal tumors with loss of H3K27me3 had shorter survival (56.12 \pm 6.67 months vs $166.17 \pm 18.88$ months, $P=0.011)(\mathbf{a})$. Figure $5 \mathbf{b}$ showed that the loss of H3K27me3 expressions, with the endothelia serving as an inner positive control

reported that the RELA fusions were related to cellularity, cellular atypia and necrosis. Malgulwar Prit Benny et al. hypothesized that RELA fusions leaded to the increased vascularity and clear cells due to upregulation of VEGF secondary by increased NF- $\mathrm{KB}$ signaling [24]. Figarella-Branger D showed that pathological NF-kB activation by this mechanism characterized human Supratentorial clear cell ependymomas with branching capillaries, which therefore represent a subset of ST-EPN-RELA. The aggressive pathological features related to RELA fusions may be induced by the aberrant NF-kB signaling, and the mechanism required further exploration.

CCND1 amplification has been reported in many cancers, including breast cancer, esophageal cancer, laryngeal, and lung cancers [25-30]. Whole gene sequencing of ependymomas identified CCND1 amplifications in some cases [9]. However, the clinical significance of CCND1 amplification is not well established. We detected CCND1 amplification in our clinical samples and found that CCND1 amplification was related to a shorter survival time especially in the intracranial cases. The survival trend indicated that CCND1 might cause malignancy in ependymomas.

H3K27me3 is a critical mark for stem cell maintenance and is mediated by EZH2, which is a member of the polycomb group (PcG) family [15]. H3K27 trimethylation plays a role in the repression of lineage regulatory genes during pluripotency in embryonic stem cells [31]. Furthermore, H3K27me3 is known to affect pathogenesis in H3F3A K27 M m DNA methylation [32]. Global loss of H3K27 trimethylation may contribute to pediatric GBM by affecting differentiation pathways [16]. H3K27me3 can also be used as a surrogate for DNA methylation; a reduction of H3K27me3 corresponds to EPN-PFA and suggests that the patients need post-surgery therapy and have a worse survival status [15]. In our study, we found that H3K27me3 could also be used as a prognostic marker for ependymal tumors. Therefore, our study suggests that the reduction of H3K27me3 may also participate in the epigenetic process in ependymal tumors.

\section{Conclusions}

In this study of our single institutional series, the prognostic factors of ependymal tumors included conventional pathological features such as Ki67 index and newly discovered molecular markers including RELA gene fusion and H3K27me3. Ependymal tumor grading could be classified by pathological characteristics, and the combination of traditional pathological parameters and molecular approaches could provide a better evaluation of ependymal tumors.

\section{Abbreviations}

CCND1: Cylclin D1; EP-PFA: Ependymomas posterior fossa group A; EPPFB: Ependymomas posterior fossa group B; EZH2: Enhancer of zeste homolog 2; FISH: Fluorescence in situ hybridization; GBM: Glioblastoma multiform; H3K27me3: Histone H3 lysine 27 trimethylation;

IHC: Immunohistochemical; L1CAM: L1 cell adhesion molecule; NFKB: Nuclear factor kappa B; RELA: v-relavian reticuloendotheliosis viral oncogene homolog A; ST-EPN: Supratentorial ependymomas; WHO: World Health Organization

\section{Acknowledgements}

Not applicable.

\section{Authors' contributions}

SYX and SK performed research and analyzed data; WMH and FW analyzed data; YSC and JW performed experimental; JZ and ZPC Design the research. All authors read and approved the final manuscript. 


\section{Funding}

This study was supported by grants from the grants as showed below: National Natural Science Funds of China (NSFC) (81372685 and 81872059) and National Basic Research Program of China (973) (2015CB755505) supported the data collection, the National High Technology Research and Development Program of China (863) (2012AA02A508) supported our study design, Guangzhou Science Technology and Innovation Project (201508020125) supported our study design, Science and Technology Planning Project (2016A020213004) and Natural Science Funds of Guangdong Province (S2013040012894) supported our study analysis, NSFC (81572479 and 81772677) and Natural Science Foundation of Guangdong Province (2016A030313309) supported the data interpretation, and NSFC (81772675) supported the manuscript written.

\section{Availability of data and materials}

The authenticity of this article has been validated by uploading the key raw data onto the Research Data Deposit public platform (www.researchdata.org.cn), with the approval RDD number as RDDB2019000580.

\section{Ethics approval and consent to participate}

The written consents were obtained from the patients or the relatives of patients. The study was approved by the Ethics Committee of the Sun Yatsen University Cancer Center.

\section{Consent for publication}

Not applicable.

\section{Competing interests}

The authors declare that they have no competing interests.

\section{Author details}

'Department of Pathology, Sun Yat-sen University Cancer Center, State Key Laboratory of Oncology in South China, Collaborative Innovation Center for Cancer Medicine, Guangzhou 510060, China. 'Department of Neurosurgery/ Neuro-oncology, Sun Yat-sen University Cancer Center, State Key Laboratory of Oncology in South China, Collaborative Innovation Center for Cancer Medicine, 651 Dongfeng Rd. East, Guangzhou 510060, China. ${ }^{3}$ Department of Molecular Diagnostics, Sun Yat-sen University Cancer Center, Collaborative Innovation Center for Cancer Medicine, Guangzhou 510060, China.

Received: 6 January 2019 Accepted: 24 June 2019

\section{Published online: 19 July 2019}

\section{References}

1. Godfraind C, et al. Distinct disease-risk groups in pediatric supratentorial and posterior fossa ependymomas[J]. Acta Neuropathol. 2012;124(2):247-57.

2. Pajtler KW, et al. Molecular classification of ependymal tumors across all CNS compartments, histopathological grades, and age groups[J]. Cancer Cell. 2015;27(5):728-43.

3. Tihan $\mathrm{T}$, et al. The prognostic value of histological grading of posterior fossa ependymomas in children: a Children's oncology group study and a review of prognostic factors[J]. Mod Pathol. 2008;21(2):165-77.

4. Bouffet $E$, et al. Intracranial ependymomas in children: a critical review of prognostic factors and a plea for cooperation[J]. Med Pediatr Oncol. 1998; 30(6):319-29; discussion 329-31.

5. Ellison DW, et al. Histopathological grading of pediatric ependymoma: reproducibility and clinical relevance in European trial cohorts[J]. J Negat Results Biomed. 2011;10(7).

6. Figarella-Branger $D$, et al. Prognostic factors in intracranial ependymomas in children[J]. J Neurosurg. 2000;93(4):605-13.

7. Kilday JP, et al. Pediatric ependymoma: biological perspectives[J]. Mol Cancer Res. 2009;7(6):765-86.

8. Korshunov A, et al. Molecular staging of intracranial ependymoma in children and adults[J]. J Clin Oncol. 2010;28(19):3182-90.

9. Parker $\mathrm{M}$, et al. C11 orf95-RELA fusions drive oncogenic NF-kappaB signalling in ependymoma[J]. Nature. 2014;506(7489):451-5.

10. DiDonato JA, Mercurio F, Karin M. NF-kappaB and the link between inflammation and cancer[J]. Immunol Rev. 2012;246(1):379-400.

11. Wang $L$, et al. IL-2Ralpha up-regulation is mediated by latent membrane protein 1 and promotes lymphomagenesis and chemotherapy resistance in natural killer/T-cell lymphoma[J]. Cancer Commun (Lond). 2018;38(1):62.
12. Baud V, Karin M. Is NF-kappaB a good target for cancer therapy? Hopes and pitfalls[J]. Nat Rev Drug Discov. 2009;8(1):33-40.

13. Kiefel $\mathrm{H}$, et al. Linking L1CAM-mediated signaling to NF-kappaB activation[J]. Trends Mol Med. 2011;17(4):178-87.

14. Hinz $M$, et al. NF-kappaB function in growth control: regulation of cyclin D1 expression and G0/G1-to-S-phase transition[J]. Mol Cell Biol. 1999;19(4):2690-8.

15. Panwalkar $P$, et al. Immunohistochemical analysis of H3K27me3 demonstrates global reduction in group-a childhood posterior fossa ependymoma and is a powerful predictor of outcome[J]. Acta Neuropathol. 2017;134(5):705-14.

16. Bender $\mathrm{S}$, et al. Reduced H3K27me3 and DNA hypomethylation are major drivers of gene expression in K27M mutant pediatric high-grade gliomas[J]. Cancer Cell. 2013;24(5):660-72.

17. Shaoyan $X$, et al. Downregulation of EIF4A2 in non-small-cell lung cancer associates with poor prognosis[J]. Clin Lung Cancer. 2013;14(6):658-65.

18. Perry A. Pathology of low-grade gliomas: an update of emerging concepts[J]. Neuro-Oncology. 2003;5(3):168-78.

19. Komori T. The $2016 \mathrm{WHO}$ classification of Tumours of the central nervous system: the major points of revision. [J] Neurol Med Chir (Tokyo). 2017; 57(7):301-11.

20. Coons, S.W., P.C. Johnson, and D.K. Pearl, The prognostic significance of Ki-67 labeling indices for oligodendrogliomas[J]. Neurosurgery, 1997.41 (4): 878-884; discussion 884-5

21. Preusser $M$, et al. Ki67 index in intracranial ependymoma: a promising histopathological candidate biomarker[J]. Histopathology. 2008;53(1):39-47.

22. Gessi $M$, et al. Role of immunohistochemistry in the identification of Supratentorial C11ORF95-RELA fused Ependymoma in routine neuropathology[J]. Am J Surg Pathol. 2017.

23. Figarella-Branger $D$, et al. Supratentorial clear cell ependymomas with branching capillaries demonstrate characteristic clinicopathological features and pathological activation of nuclear factor-kappaB signaling[J]. NeuroOncology. 2016;18(7):919-27.

24. Malgulwar PB, et al. C11 orf95-RELA fusions and upregulated NF-KB signalling characterise a subset of aggressive supratentorial ependymomas that express L1CAM and nestin[J]. J Neuro-Oncol. 2018;138(1):29-39.

25. Gautschi O, et al. Cyclin D1 in non-small cell lung cancer: a key driver of malignant transformation[J]. Lung Cancer. 2007:55(1):1-14.

26. Jiang $W$, et al. Amplification and expression of the human cyclin D gene in esophageal cancer[J]. Cancer Res. 1992;52(10):2980-3.

27. Buckley MF, et al. Expression and amplification of cyclin genes in human breast cancer[J]. Oncogene. 1993;8(8):2127-33.

28. Gillett $C$, et al. Amplification and overexpression of cyclin D1 in breast cancer detected by immunohistochemical staining[J]. Cancer Res. 1994; 54(7):1812-7.

29. Hosokawa Y, et al. Absence of cyclin D1/PRAD1 point mutations in human breast cancers and parathyroid adenomas and identification of a new cyclin D1 gene polymorphism[J]. Cancer Lett. 1995;93(2):165-70.

30. Betticher DC, et al. Prognostic significance of CCND1 (cyclin D1) overexpression in primary resected non-small-cell lung cancer[J]. Br J Cancer. 1996;73(3):294-300.

31. Vastenhouw NL, Schier AF. Bivalent histone modifications in early embryogenesis[J]. Curr Opin Cell Biol. 2012;24(3):374-86.

32. Hagarman JA, et al. Coordinate regulation of DNA methylation and H3K27me3 in mouse embryonic stem cells[J]. PLoS One. 2013;8(1):e53880.

\section{Publisher's Note}

Springer Nature remains neutral with regard to jurisdictional claims in published maps and institutional affiliations. 